\title{
Usefulness of Thrombin Generation
}

\author{
Armando Tripodi ${ }^{1}$ \\ ${ }^{1}$ IRCCS Ca' Granda Ospedale Maggiore Policlinico Foundation, Angelo \\ Bianchi Bonomi Hemophilia and Thrombosis Center and Fondazione \\ Luigi Villa, Milan, Italy
}

Hämostaseologie 2020;40:509-514. Address for correspondence Armando Tripodi, PhD, Via Pace 9,
Milano, 20122, Italy (e-mail: Armando.tripodi@unimi.it).

\begin{abstract}
Keywords

- arterial thrombosis

- coagulation factors

- Hemostasis

Thrombin generation (TG) is a global coagulation procedure meant to continuously monitor thrombin formation and decay upon exposure of platelet-poor plasma to exogenous triggers such as tissue factor, phospholipids, and calcium chloride. The procedure can also be performed in platelet-rich plasma by omitting exogenous phospholipids. TG is thought to mimic more than other coagulation procedures the process that occurs in vivo. Over the years, TG has been used to investigate coagulation mechanisms, which were not completely understood, or to investigate hyper- or hypocoagulability in clinical conditions known to be at increased risk of thrombosis or hemorrhage, respectively. More recently, TG has been employed as a laboratory tool to assess the risk of recurrent venous thromboembolism or to assess the risk of arterial thromboembolism in specific clinical settings or in the general population. The article reviews the value and limitations of TG.
\end{abstract}

\section{Introduction}

Thrombin is the key enzyme of coagulation, responsible for the fibrinogen to fibrin conversion. Beside this paramount function, thrombin mediates many other mechanisms, which are instrumental for hemostasis, such as the activation of platelets, protein C, factors VIII, V, XI, XIII, and the thrombin activatable fibrinolysis inhibitor. Furthermore, thrombin mediates mechanisms which are apparently well beyond hemostasis. For example, thrombin regulates the vascular tone and permeability, the inflammatory response, ${ }^{1,2}$ the liver parenchymal extinction, subsequent to the progression of fibrosis in patients with cirrhosis, ${ }^{3,4}$ and many others. It is therefore not surprising that the quantification of thrombin generation (TG) in clotting blood or plasma has attracted the attention of researchers over time.

In the past, thrombin was measured as its ability to clot whole blood or plasma in the early global procedures of coagulation, namely prothrombin time (PT) and activated partial thromboplastin time (APTT). Much more attention to the TG, evaluated with different procedures, came about in the early 1950s when MacFarlane and Biggs ${ }^{5}$ performed experiments of in vitro TG measurement. They sought to activate coagulation in whole blood or plasma by triggers such as cephaline or tissue extracts from human or animal origin and calcium chloride. Following the activation, small amounts of the clotting mixture were transferred at sequential time points into a series of test tubes containing a fibrinogen solution. The clotting times of the fibrinogen solution were inversely related to the thrombin activity generated at specific time points and were used to interpolate thrombin activity from a dose-response curve. Thrombin activity was then used to construct TG curves generated over time. The typical pattern was described by the increasing thrombin activity soon after the coagulation ignition, followed by a peak and declining activity as soon as the naturally occurring anticoagulant mechanisms started to inhibit TG. However, the procedure was soon abandoned because it was deemed too complex for routine use. Many years later it was resumed by Hemker et $\mathrm{al}^{6}{ }^{6}$ who made substantial changes (reviewed in Tripodi ${ }^{7}$ ). The endpoint for the measurement of thrombin activity switched from clotting fibrinogen to cleavage of a chromogenic substrate. This allowed the procedure to be performed in clinical chemistry received

May 4, 2020

accepted after revision

June 15,2020 (c) 2020 Georg Thieme Verlag KG Stuttgart · New York
DOI https://doi.org/

10.1055/a-1200-0417. ISSN 0720-9355. 
analyzers. However, one of the limitations of the procedure was the presence of fibrin that was generated during clot formation. The potential interference brought about by the turbidity of fibrin on the analyzer optical system was resolved either by defibrination of the plasma prior to testing or by including in the system an inhibitor to fibrin polymerization. Other key changes have been introduced over the years, namely the substitution of the chromogenic in favor of a fluorogenic substrate and the use of a computer software coupled with a dedicated fluorimeter. The former allowed the procedure to be performed in platelet-poor or plateletrich plasma without defibrination and the latter allowed the continuous monitoring of TG in a completely automated fashion for many samples at the same time. Currently, the procedure is designed to operate on a microplate-based assay and has been extensively used over the last two decades as a multipurpose laboratory tool. A new TG procedure has been recently introduced (i.e., ST Genesia), which is designed to assess TG under strict control of temperature and volumes, ensuring easy and reliable performance even in less specialized laboratories. ${ }^{8}$ The practice will inform on its suitability as a laboratory tool for patient management. Finally, a TG procedure for testing whole blood has been developed, but not yet thoroughly evaluated. ${ }^{9}$

\section{Unresolved Issues}

Although there are still unresolved issues concerning the choice and standardization of reagents, assay reproducibility, and result expression, TG is now ready for search of indications stemming from clinical trials. Currently, there are different commercial TG methods under different brand names and their results are hardly comparable. ${ }^{10}$ This means that results obtained in patient plasma can be hardly compared with the traditional upper and lower limits of the reference interval, as it occurs for many other laboratory tests. Until these issues will be resolved, it should be recommended to perform case-control studies in which samples from controls undergo exactly the same testing procedure as the patient samples. The above design should be recommended especially whenever the procedure is performed in the presence of thrombomodulin (TM; meant to optimally activate protein C) or corn trypsin inhibitor (CTI; meant to quench the accidental activation of contact coagulation factors). The addition of TM or CTI may variably affect the results of TG parameters, depending on the assay condition and/or the batch or reagents. It is therefore important that results from patients and controls be confronted under the same experimental conditions.

\section{Significance of the Thrombin Generation Parameters}

The composite pattern of TG is expressed by the thrombogram and it is defined (among others) by the following parameters ( - Fig. 1 and -Table 1).(1) The lag time, which is a measure of the time (minutes) elapsing from the ignition of coagulation to the initial thrombin appearance. The lag time can be regarded as the traditional plasma clotting time and is expected to be

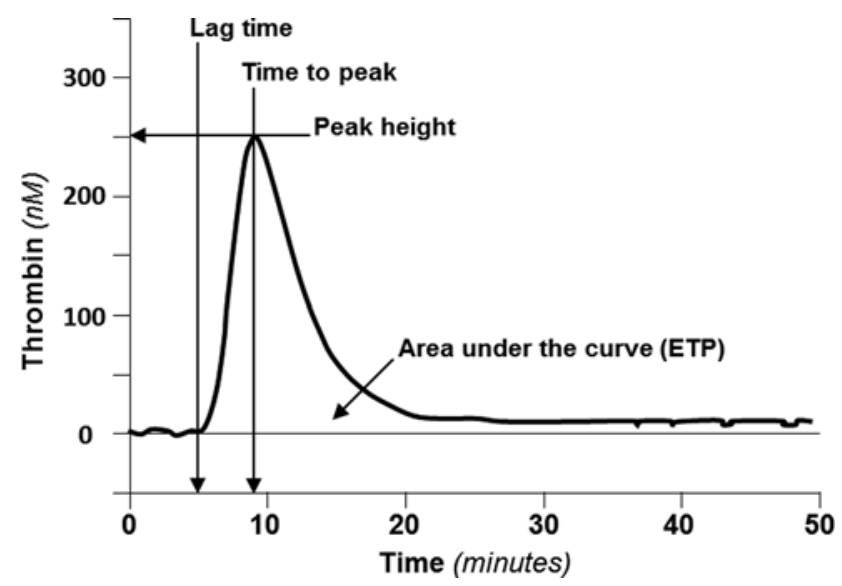

Fig. 1 Main parameters of the thrombin generation curve (see the text for more details).

shortened or prolonged in those conditions characterized by hyper- or hypocoagulability. (2) The peak thrombin, which represents the highest thrombin concentration (nM) that can be reached for the testing plasma under the specified experimental conditions. Peak thrombin is expected to be high or low in those conditions characterized by hyper- or hypocoagulability. (3)The time to reach the peak, which is a measure of the time (minutes) needed to reach the peak. This parameter is expected to be shortened or prolonged in those conditions characterized by hyper- or hypocoagulability. (4) The area under the curve, also called endogenous thrombin potential (ETP), which represents the net amount of thrombin that the test plasma can generate under the experimental conditions as a result of the two opposing coagulation drivers operating in plasma (i.e., pro- and anticoagulants). ETP is expected to be low or high in those conditions characterized by hypo- or hypercoagulability.

\section{Test Plasma and Reagents Needed for Thrombin Generation}

The preparation of test plasma and reagents used for TG may considerably affect the results of the procedure. ${ }^{11}$ Therefore, great care should be exerted for their appropriate definition.

\section{Test Plasma}

Residual platelets in plasma, especially after freezing-thawing, may have considerable detrimental effects on TG parameters, as platelets upon fragmentation expose negatively charged phospholipids, which may considerably increase the procoagulant driving force of the system. Plasma for TG should be prepared under careful standardized centrifugation to get residual platelets as low as possible. Double centrifugation has been advocated, but cannot be used on a regular basis, as it is not the standard practice in general laboratories that work by automated procedures. Furthermore, many samples prepared for general purpose via the standard centrifugation cannot be later used for TG. An acceptable compromise would be the blood centrifugation at 3,000 $\mathrm{g}$ for 15 minutes (controlled room temperature). This procedure would allow getting plasma with minimal residual platelets. Supernatant plasma 
can be stored frozen for later use, if the storage temperature is $-70^{\circ} \mathrm{C}$ or below. Frozen plasma must be thawed rapidly (immersion in a water bath at $37^{\circ} \mathrm{C}$ for $1-2$ minutes) and homogenized by gentle inversion before testing. In special situations, when one wishes to investigate the role played by the platelet procoagulant activity, platelet-rich plasma can be used with addition of tissue factor, but not of exogenous negatively charged phospholipids. ${ }^{12}$ On these occasions, strict standardization of platelet counts in platelet-rich plasma is paramount and it is also recommended to run control samples from healthy subjects along with patient samples.

\section{Triggers}

Coagulation ignition is performed by small amounts of tissue factor and negatively charged phospholipids. At variance with the PT and APTT, the concentrations of these triggers should be maintained as low as possible to mimic in vitro as much as possible the conditions operating in vivo. The most used tissue factor (final) concentrations are 1 or $5 \mathrm{pM}$ and the source is (diluted) thromboplastin from relipidated recombinant tissue factor. Higher concentrations can be used in special situations (heparins or other anticoagulants) when the procoagulant force of the plasma is expected to be relatively small. Negatively charged phospholipids are usually a blend of synthetic phospholipids at concentrations ranging from 1 to $4 \mu \mathrm{M}$. It should be realized that the source and concentration of triggers may have considerable effect on TG parameters and therefore strict adherence to standardization and/or case-control studies should be considered to compare results over time.

\section{Addition of other Agents}

TG can be measured in the presence of $\mathrm{TM}^{13} \mathrm{TM}$ is the physiological activator of protein $\mathrm{C}$, which is relatively abundant in endothelial cells and much less in plasma. Therefore, TG does not fully account for the anticoagulant activity of protein C, as this naturally occurring anticoagulant cannot be optimally activated in the absence of TM. This may be detrimental in some clinical conditions where protein $C$ is greatly reduced. Typical examples are the congenital deficiency of protein $C$ as well as its acquired deficiency due to liver cirrhosis (see below). In both conditions, the relevant anticoagulant driving force of protein $\mathrm{C}$ would be undetected in the absence of TM. Therefore, the addition of soluble TM in the test system is beneficial for the proper assessment of the balance between pro- and anticoagulants. However, the optimal amount of TM to be added to the test system is difficult to determine as in vivo TM is located on endothelial cells and its density/concentration is not accurately known. A pragmatic solution could be to add an amount of TM, which is able to reduce the ETP of the pooled normal plasma by $50 \%$. The correspondent TM concentration to achieve this reduction varies from lot to lot and should be evaluated for each lot of TM used over time. ${ }^{13}$ Activated protein C (APC) can also be added to the TG system to help in increasing the sensitivity to acquired plasma APC resistance. ${ }^{14}$

Another important component of the TG procedure could be CTI. CTI is able to inhibit the contact phase of coagulation, thus avoiding the contribution to TG that could come about from an accidental activation of contact coagulation factors (e.g., factor XII, pre-kallikrein, or high-molecular-weight kininogen). This activation may be variably occurring when plasma samples are stored frozen for later use. CTI should be added directly in the tube used for blood collection, ${ }^{15}$ as later addition to plasma could be ineffective. The value of adding CTI has been debated in the literature (reviewed in Tripodi et $\mathrm{al}^{16}$ ) and was abandoned because it deemed difficult in practice and because of the relatively high cost. Perhaps, as mentioned above a more pragmatic solution could be running casecontrol studies in plasma without addition of CTI, assuming that contact activation (if any) would affect the control and patient samples to the same extent.

\section{Result Expression}

Results of TG parameters are usually expressed as time (minutes) for lag time and time to peak, nM thrombin for peak, and $\mathrm{nM}$ thrombin $\times$ minutes for the area under the curve (ETP). These result expressions are poorly standardized and may vary owing to small methodological variations and/or changing reagent lots that may occur over time. Attempts to standardize this step have been done by expressing results relative to a pooled normal plasma taken as a standard and tested along with the patient plasma. ${ }^{17}$ International standards have not yet been provided. As mentioned, the safest way to proceed for a fair comparison of results obtained over time is running case-control studies.

\section{Use of Thrombin Generation}

As mentioned, over the last two decades TG attracted the attention of many. Searching PubMed with the key word "thrombin generation" yields nearly 7,500 items. Close examination reveals that they are dealing with a certain number of fields for which TG was successfully used. Not all of them are however related to significant applications on the direct management of patients. In the following paragraphs, I discuss the merits and limitations on the application of the procedure (-Table 2).

\section{Investigation of Mechanisms of Thrombogenesis}

As mentioned, thrombin plays a key role in clot formation in vitro and in vivo. Therefore, the use of TG may help in understanding the process of thrombogenesis in many clinical conditions that were for long time associated with an increased risk of thrombosis, but for which there was no clear demonstration of plasma hypercoagulability. This is not surprising as the conventional procedures used to assess coagulation such as the global tests PT and APTT or the measurement of the individual components of the coagulation balance (either pro-, anticoagulants, or both) present with obvious limitations. PT and APTT are static tests that are unable to account for the entire process from TG to decay, based on the two opposing drivers (pro- and anticoagulants). On the other hand, the measurement of the individual components (pro- and anticoagulants) can tell us whether or not one or more individual components are deranged, but not if the balance between the 
two is or is not perturbed. This may occur especially in clinical conditions associated with acquired hemostasis disorders when the activities of both pro- and anticoagulants are variably reduced or increased. Indeed, TG has been successfully used to unveil plasma hypercoagulability in many conditions that have for long time been associated with an increased risk of thrombosis, especially venous thrombosis. Among them, one can mention diabetes, ${ }^{18}$ obesity, ${ }^{19}$ Cushing syndrome, ${ }^{20}$ fatty liver, ${ }^{21,22}$ inflammatory bowel disease, ${ }^{23}$ and many others for which it has been shown that TG is increased in spite of the fact that PT, APTT, and the individual components of coagulation are near normal. Furthermore, TG procedures performed in the presence/absence of TM has been instrumental in dismantling the old dogma that depicted liver cirrhosis as the prototype of the acquired hemorrhagic disease. ${ }^{24,25}$ Thanks to TG, we now know that cirrhosis is associated with an acquired coagulopathy characterized by rebalanced but unstable coagulation that (depending on the circumstances and risk factors) may tip toward thrombosis or hemorrhage. ${ }^{26}$ TG has also been used to help in understanding mechanisms of thrombogenesis in special situations. For example, Miyawaki et $\mathrm{al}^{27}$ have used TG to investigate a prothrombin variant associated with thrombosis. They found that the start tail (i.e., duration of thrombin-generation activity), assessed from the area under the TG curve, was impaired (extended) in the carriers of the mutation.

\section{Investigation of Hemorrhagic Conditions}

TG helped in understanding the relative contribution of coagulation factor to TG and fibrin formation. A situation where the basic tests of coagulation (PT and APTT) or the measurement of the individual coagulation factors cannot help, as coagulation is a tight integrated system that requires full cooperation of many factors to generate thrombin and to convert fibrinogen into fibrin. ${ }^{28}$

\section{Monitoring Procoagulant Agents}

A good example in this context is hemophilia. Up to $30 \%$ of hemophiliacs may develop during their life specific inhibitors to factor VIII or IX. When the inhibitor titer is relatively high, they cannot be treated with conventional coagulation factor concentrates and benefit from infusion of prohemostatic agents (either activated prothrombin complex concentrates or recombinant activated factor VII) collectively called bypassing agents. Monitoring such drugs by the postinfusion measurement of the individual coagulation factor VIII or IX does not make sense as bypassing agents exert their hemostatic efficacy with little modification of these factors. TG procedures would instead be the candidate monitoring systems. $^{29}$ TG procedures have also been found useful in the context of hemophilia treatment in special conditions. ${ }^{30}$

\section{Evaluation of the Risk Recurrence of Venous Thromboembolism}

The risk of recurrent venous thromboembolism (VTE) after a first episode is relatively high, especially for unprovoked events. The optimal duration of the secondary prophylaxis in these patients is paramount and should be established by careful considerations of the benefit/risk ratio, which include clinical as well as laboratory considerations. Among the latter, D-dimer has been established over the last two decades as one of the laboratory tools that can be used to assess the risk of VTE recurrence after discontinuation of anticoagulation. ${ }^{31}$ The TG procedure also proved to be a good predictor of recurrent VTE $\mathrm{V}^{32,33}$ and can therefore be used alone or in combination with D-dimer ${ }^{34}$ to assess the risk of recurrence and to make decision on the optimal duration of secondary prophylaxis.

\section{Monitoring Patients on Antithrombotic Drugs}

Parenteral or oral antithrombotic drugs (e.g., heparins, vitamin $\mathrm{K}$ antagonists, or direct oral anticoagulants) share the same principle. Whatever their mechanism of action, they ultimately act by downregulating TG. It is therefore obvious that TG procedures are suitable to assess their antithrombotic capacity. ${ }^{35}$ It should however be recognized that before TG can be used in this context, it should be determined whether it is superior to the other laboratory tools (e.g., the international normalized ratio, the APTT, or the individual measurement of the direct oral anticoagulants) which are currently used to monitor antithrombotic drugs.

\section{Investigation of the Risk of Thrombosis in the General Population}

Thrombosis is cumulatively considered as one of the most important health burdens, especially in western countries. Therefore, the quest for laboratory tools able to identify those apparently healthy individuals, who will later develop thrombotic events, has attracted over the years the attention of epidemiologists, clinicians, and laboratory operators. In this context, PT and APTT play a minor role, although shortened APTT proved to be associated with the risk of VTE in retrospective case-control studies. ${ }^{36}$ TG has been investigated as a global laboratory tool as a predictor of the risk of arterial thrombosis in studies of healthy subjects. ${ }^{37,38}$ In particular, the results of the Gutenberg Health Study ${ }^{38}$ that investigated 5,000 healthy subjects, who were followed up to 9 years, showed that the hypercoagulability detected by TG is associated with overall mortality. This is a promising result, although it does not appear applicable to assess the risk in individual patients.

\section{Concluding Remarks}

Thrombogenesis as well as the mechanisms of action of the many antithrombotic drugs operates through the enhancement or reduction of TG in plasma. It is therefore not surprising that the TG procedures described by the pioneer work of MacFarlane and Biggs and later by Hemker and coworkers attracted the attention of physicians, epidemiologists, and laboratory operators. Presently, TG procedures play a central role in the laboratory investigation of the mechanisms of thrombogenesis in many clinical conditions, which were poorly understood until recently. Whether they can also be used in the daily management of patients affected by hemorrhage or thrombosis requires further investigations. 
Table 1 Main parameters of thrombin generation and their significance

\begin{tabular}{|l|l|}
\hline Parameter & Significance \\
\hline Lag time & Short or long lag time, suggest hyper- or hypocoagulability \\
\hline Peak & High or low peak, suggest hyper- or hypocoagulability \\
\hline Time to peak & Short or long time to peak, suggest hyper- or hypocoagulability \\
\hline Area under the curve (ETP) & High or low ETP, suggest hyper- or hypocoagulability \\
\hline
\end{tabular}

Abbreviation: ETP, endogenous thrombin potential.

Table 2 Potential applications of thrombin generation (see the text for more information)

\begin{tabular}{|l|l|}
\hline Application & Examples \\
\hline $\begin{array}{l}\text { Investigation of mechanisms } \\
\text { of thrombogenesis }\end{array}$ & $\begin{array}{l}\text { Liver cirrhosis, diabetes, obesity, Cushing syndrome, } \\
\text { fatty liver, inflammatory bowel disease, and others }\end{array}$ \\
\hline Investigation of hemorrhagic conditions & Hemophilia and rare hemorrhagic coagulopathies \\
\hline Monitoring procoagulant agents & $\begin{array}{l}\text { Hemophilia with and without inhibitors (especially treatment } \\
\text { with bypassing agents) }\end{array}$ \\
\hline Venous thromboembolism & Evaluation of the risk recurrence of venous thromboembolism \\
\hline Monitoring antithrombotic drugs & Vitamin K antagonists, heparins, direct (oral or parenteral) anticoagulants \\
\hline Arterial thromboembolism & Investigation of the risk of thrombosis in the general population \\
\hline
\end{tabular}

Conflict of Interest

None declared.

\section{References}

1 Garcia JG, Pavalko FM, Patterson CE. Vascular endothelial cell activation and permeability responses to thrombin. Blood Coagul Fibrinolysis 1995;6(07):609-626

2 Siller-Matula JM, Schwameis M, Blann A, Mannhalter C, Jilma B. Thrombin as a multi-functional enzyme. Focus on in vitro and in vivo effects. Thromb Haemost 2011;106(06):1020-1033

3 Wanless IR, Wong F, Blendis LM, Greig P, Heathcote EJ, Levy G. Hepatic and portal vein thrombosis in cirrhosis: possible role in development of parenchymal extinction and portal hypertension. Hepatology 1995;21(05):1238-1247

4 Bitto N, Liguori E, La Mura V. Coagulation, microenvironment and liver fibrosis. Cells 2018;7(08):85

5 MacFarlane RG, Biggs R. A thrombin generation test; the application in haemophilia and thrombocytopenia. J Clin Pathol 1953;6 (01):3-8

6 Hemker HC, Willems GM, Béguin S. A computer assisted method to obtain the prothrombin activation velocity in whole plasma independent of thrombin decay processes. Thromb Haemost 1986;56(01):9-17

7 Tripodi A. The history of phenotypic testing in thrombosis and hemostasis. Semin Thromb Hemost 2008;34(07):585-592

8 Douxfils J, Morimont L, Bouvy C, et al. Assessment of the analytical performances and sample stability on ST Genesia system using the STG-DrugScreen application.J Thromb Haemost 2019;17(08): 1273-1287

9 Ninivaggi M, Apitz-Castro R, Dargaud Y, de Laat B, Hemker HC, Lindhout $\mathrm{T}$. Whole-blood thrombin generation monitored with a calibrated automated thrombogram-based assay. Clin Chem 2012;58(08):1252-1259

10 de Laat-Kremers RMW, Ninivaggi M, Devreese KMJ, de Laat B. Towards standardization of thrombin generation assays: inventory of thrombin generation methods based on results of an International Society of Thrombosis and Haemostasis Scientific
Standardization Committee survey. J Thromb Haemost 2020 (epub ahead of print) . Doi: 10.1111/jth.14863

11 Chantarangkul V, Clerici M, Bressi C, Giesen PL, Tripodi A. Thrombin generation assessed as endogenous thrombin potential in patients with hyper- or hypo-coagulability. Haematologica 2003; 88(05):547-554

12 Tripodi A, Primignani M, Chantarangkul V, et al. Thrombin generation in patients with cirrhosis: the role of platelets. Hepatology 2006;44(02):440-445

13 Tripodi A. Detection of procoagulant imbalance. Modified endogenous thrombin potential with results expressed as ratio of values with-to-without thrombomodulin. Thromb Haemost 2017;117 (05):830-836

14 Curvers J, Thomassen MC, Nicolaes GA, et al. Acquired APC resistance and oral contraceptives: differences between two functional tests. Br J Haematol 1999;105(01):88-94

15 Luddington R, Baglin T. Clinical measurement of thrombin generation by calibrated automated thrombography requires contact factor inhibition. J Thromb Haemost 2004;2(11):1954-1959

16 Tripodi A, Chantarangkul V, Mancuso ME, Lemma L, Clerici M, Santagostino E. Comparison of thrombin generation for pairedplatelet-rich plasma collected with and without corn trypsin inhibitor from hemophiliacs treated with factor-VIII inhibitor bypassing agents. J Thromb Haemost 2012;10(04):716-719

17 Dargaud Y, Luddington R, Gray E, et al. Standardisation of thrombin generation test-which reference plasma for TGT? An international multicentre study. Thromb Res 2010;125(04):353-356

18 Tripodi A, Branchi A, Chantarangkul V, et al. Hypercoagulability in patients with type 2 diabetes mellitus detected by a thrombin generation assay. J Thromb Thrombolysis 2011;31(02): 165-172

19 Tripodi A, Primignani M, Badiali S, et al. Body mass index reduction improves the baseline procoagulant imbalance of obese subjects. J Thromb Thrombolysis 2019;48(01):52-60

20 Tripodi A, Ammollo CT, Semeraro F, et al. Hypercoagulability in patients with Cushing disease detected by thrombin generation assay is associated with increased levels of neutrophil extracellular trap-related factors. Endocrine 2017;56(02):298-307 
21 Tripodi A, Fracanzani AL, Chantarangkul V, Primignani M, Fargion S. Procoagulant imbalance in patients with non-alcoholic fatty liver disease. J Hepatol 2017;66(01):248-250

22 Potze W, Siddiqui MS, Boyett SL, et al. Preserved hemostatic status in patients with non-alcoholic fatty liver disease. J Hepatol 2016; 65(05):980-987

23 Saibeni S, Saladino V, Chantarangkul V, et al. Increased thrombin generation in inflammatory bowel diseases. Thromb Res 2010; 125(03):278-282

24 Tripodi A, Salerno F, Chantarangkul V, et al. Evidence of normal thrombin generation in cirrhosis despite abnormal conventional coagulation tests. Hepatology 2005;41(03):553-558

25 Gatt A, Riddell A, Calvaruso V, Tuddenham EG, Makris M, Burroughs AK. Enhanced thrombin generation in patients with cirrhosis-induced coagulopathy. J Thromb Haemost 2010;8(09): 1994-2000

26 Tripodi A, Primignani M, Mannucci PM, Caldwell SH. Changing concepts of cirrhotic coagulopathy. Am J Gastroenterol 2017;112 (02):274-281

27 Miyawaki Y, Suzuki A, Fujita J, et al. Thrombosis from a prothrombin mutation conveying antithrombin resistance. $\mathrm{N}$ Engl J Med 2012;366(25):2390-2396

28 Zekavat OR, Haghpanah S, Dehghani J, Afrasiabi A, Peyvandi F, Karimi M. Comparison of thrombin generation assay with conventional coagulation tests in evaluation of bleeding risk in patients with rare bleeding disorders. Clin Appl Thromb Hemost 2014;20(06):637-644

29 Tran HT, Sørensen B, Bjørnsen S, Pripp AH, Tjønnfjord GE, Andre Holme P. Monitoring bypassing agent therapy - a prospective crossover study comparing thromboelastometry and thrombin generation assay. Haemophilia 2015;21(02):275-283

30 Dargaud Y, Lienhart A, Janbain M, Le Quellec S, Enjolras N, Negrier C. Use of thrombin generation assay to personalize treatment of breakthrough bleeds in a patient with hemophilia and inhibitors receiving prophylaxis with emicizumab. Haematologica 2018; 103(04): e181-e183

31 Palareti G, Cosmi B, Legnani C, et al; PROLONG Investigators. Ddimer testing to determine the duration of anticoagulation therapy. N Engl J Med 2006;355(17):1780-1789

32 Tripodi A, Legnani C, Chantarangkul V, Cosmi B, Palareti G, Mannucci PM. High thrombin generation measured in the presence of thrombomodulin is associated with an increased risk of recurrent venous thromboembolism. J Thromb Haemost 2008;6 (08):1327-1333

33 van Hylckama Vlieg A, Baglin CA, Luddington R, MacDonald S, Rosendaal FR, Baglin TP. The risk of a first and a recurrent venous thrombosis associated with an elevated D-dimer level and an elevated thrombin potential: results of the THE-VTE study. J Thromb Haemost 2015;13(09):1642-1652

34 Eichinger S, Hron G, Kollars M, Kyrle PA. Prediction of recurrent venous thromboembolism by endogenous thrombin potential and D-dimer. Clin Chem 2008;54(12):2042-2048

35 Tripodi A, Padovan L, Veena C, Scalambrino E, Testa S, Peyvandi F. How the direct oral anticoagulant apixaban affects thrombin generation parameters. Thromb Res 2015;135(06):1186-1190

36 Tripodi A, Chantarangkul V, Martinelli I, Bucciarelli P, Mannucci PM. A shortened activated partial thromboplastin time is associated with the risk of venous thromboembolism. Blood 2004;104 (12):3631-3634

37 Smid M, Dielis AW, Winkens M, et al. Thrombin generation in patients with a first acute myocardial infarction. J Thromb Haemost 2011;9(03):450-456

38 van Paridon PCS, Panova-Noeva M, van Oerle R, et al. Thrombin generation in cardiovascular disease and mortality - results from the Gutenberg Health Study. Haematologica 2019 (e-pub ahead of print) . Doi: 10.3324/haematol.2019.221655 